\title{
Strain Induced Deep Electronic States around Threading Dislocations in GaN
}

\author{
L. Lymperakis and J. Neugebauer* \\ Fritz-Haber-Institut der Max-Planck-Gesellschaft, Faradayweg 4-6, D-14195 Berlin (Dahlem), Germany \\ Fakultät für Naturwissenschaften, Universität Paderborn, Fachbereich 6-Physik, D-33095 Paderborn, Germany \\ M. Albrecht, T. Remmele, and H. P. Strunk \\ Institut für Werkstoffwissenschaften, Universität Erlangen-Nürnberg, Mikrocharakteriserung, \\ Cauerstrasse 6, D-91058 Erlangen, Germany
}

(Received 22 August 2003; published 1 November 2004)

\begin{abstract}
Combining through-focus high-resolution transmission electron microscopy and hierarchical multiscale simulations consisting of density-functional theory, analytical empirical potentials, and continuum elastic theory we demonstrate the existence of a new dislocation type in GaN. In contrast with all previously identified or suggested dislocation structures in $\mathrm{GaN}$, all core atoms are fully coordinated; i.e., no broken bonds occur, implying that the dislocation should be electrically inactive. However, as we show, the giant local strain-field around the dislocation core, in combination with the small lattice constant of $\mathrm{GaN}$, causes deep defect states and thus electrically active edge dislocations independent on the specific core structure.
\end{abstract}

Dislocations are important defects in semiconductors with strong effects on their electronic properties. A common approach to describe the effect a dislocation has on the electronic structure is to consider two separate regions: (i) the core region where bonds are broken, i.e., localized dangling bonds are present, and (ii) the local strain field around the core where atoms are fully (bulklike) coordinated but slightly displaced with respect to their bulk positions. The core region-due to the presence of localized dangling bond states - is expected to induce deep states within the band gap (making the dislocation electrically active) while the strained region around the dislocation is assumed to give rise to only shallow levels. Consequently, it is commonly expected that removing the core dangling bond states by reconstructing the core (e.g., by forming new bonds or rehybridization) the dislocation becomes electrically inactive. This effect is considered to be crucial for device applications: electrically inactive dislocations do not act as nonradiative recombination centers and thus have no detrimental effect on optical properties such as luminescence.

A semiconductor well known to exhibit huge dislocation densities even in device quality material is $\mathrm{GaN}$ : Typically threading dislocation densities between $10^{8}$ and $10^{10} \mathrm{~cm}^{-2}$ are observed [1]. While the origin of these defects is well understood - they are caused by the lack of a suitable, i.e., thermally and lattice matched substrate - the electronic structure of these defects is still under debate. In particular, there are contradicting experimental and theoretical reports whether threading dislocations are electrically active or not [2-10]. In the present Letter we demonstrate by a combined theoretical and experimental analysis the existence of a new type of edge dislocation structure in $\mathrm{GaN}$. A unique feature of the new structure compared to all previously identified struc- tures is that all core atoms are fully coordinated (i.e., they are, as in bulk GaN, surrounded by four anions or cations). Thus, according to the standard picture of dislocations discussed above such a dislocation should be electrically inactive. However, this is not valid for edge dislocations in $\mathrm{GaN}$ : As we show the local strain field around the dislocation core in combination with the small lattice constant of $\mathrm{GaN}$ induces in this material deep defect states.

Let us first focus on experiment. The investigated $200 \mu \mathrm{m}$ thick GaN layers were grown by hydride vapor phase epitaxy (HVPE) onto 6H-SiC substrates at a growth temperature of $1050{ }^{\circ} \mathrm{C}$ with a growth rate of about $0.06 \mathrm{~mm} / \mathrm{h}$. Transmission electron microscopy planeview samples were prepared from these crystals close to the interface between $\mathrm{SiC}$ and $\mathrm{GaN}$ by standard techniques including mechanical grinding, polishing, and $4 \mathrm{keV} \mathrm{Ar}^{+}$ion milling with a liquid nitrogen cooling stage until electron transparency was reached. Highresolution transmission electron microscopy (HR-TEM) investigations were performed in a Philips CM300 UT microscope operated at $300 \mathrm{kV}$. For each dislocation a defocus series with defocus steps of $5 \mathrm{~nm}$ was taken including at least one contrast inversion. Figures 1(a) and 1(e) show the HR-TEM pictures of the same dislocation at two defocus values $(\Delta f=-23$ and $-63 \mathrm{~nm})$. In order to identify the core structure image simulations have been performed based on the EMS program package and using standard parameters for the Philips CM 300 UT (accelerating voltage $300 \mathrm{keV}$, spherical aberration $c_{s}=$ $0.64 \mathrm{~mm}$, beam semiconvergence angle $12.6 \mathrm{mrad}$, and defocus spread $10 \mathrm{~nm}$ ). It is crucial for the comparison of experimental micrographs with contrast calculations to thoroughly determine the defocus used for imaging and local thickness of the specimen. The defocus values were 


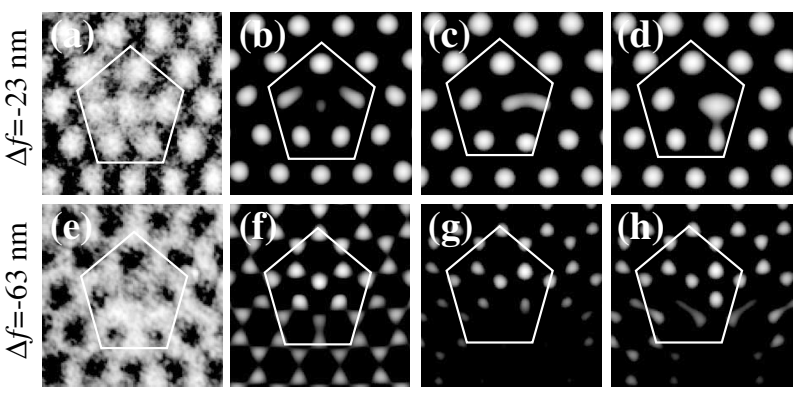

FIG. 1. Threading dislocation in $\mathrm{GaN}$ at defocus values $\Delta f=$ -23 and $\Delta f=-63 \mathrm{~nm}$ (unfiltered HR-TEM) (a),(e). Image simulations for the four-core structure (b),(f), full-core structure (c), (g), and the open structure (d),(h). The upper (lower) panel shows defocus values $\Delta f=-23 \mathrm{~nm}(\Delta f=-63 \mathrm{~nm})$. For additional experimental micrographs, see [11].

determined from a comparison of experimental diffractograms of the amorphous rim (produced due to ion milling) of the specimen with theoretical ones (calculated with the known microscope data). The thickness was determined from a comparison with contrast calculations of an undisturbed crystal. These value pairs (defocus and thickness) are then used to select the theoretical core structure images from respective defocus-thickness maps and to compare these with the experimental images. It is important for this procedure that the analyzed dislocation lies along [0001] because otherwise contrasts due to distortions may affect the evaluation.

Atomic core geometries as calculated in previous density-functional theory studies $[5,6]$ have been used as input for the image simulations: The open core also named a 5/7 ring structure [see Fig. 2(c) and respective contrast simulations in Figs. 1(d) and 1(h)]; the full core also named an eight-core structure [see Fig. 2(b) and respective contrast simulations in Figs. 1(c) and 1(g)] and the $\mathrm{Ga}$ - and $\mathrm{N}$-vacancy structure (where undercoordinated $\mathrm{Ga}$ and $\mathrm{N}$ atoms have been removed from the full core structure). Comparison of the experimental image in Fig. 1(a) with the simulated contrasts Fig. 1(c) and 1(d) shows no correspondence. Thus the "classical" core structures fail to reproduce the experimental images observed in our samples and can be ruled out as structural models. In order to identify the core structure a theoretical analysis of the atomic geometry and the energetics is crucial. A
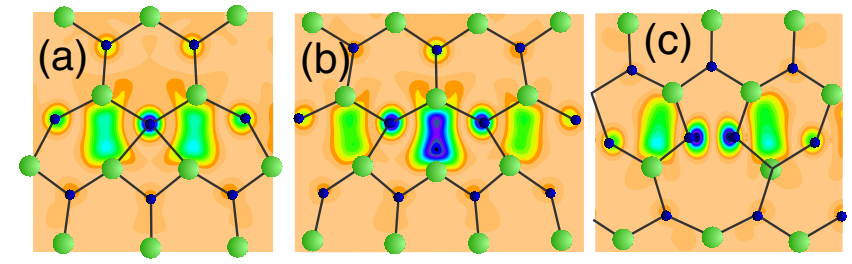

FIG. 2 (color online). Contour plots of dislocation induced deep electronic states for the (a) four-core, (b) full-core, and (c) open-core structures. Large (small) balls correspond to Ga (N) atoms. challenge in performing such calculations is the large range of relevant length scales: While the core structure itself is rather localized the strain field is significant even far away from the dislocation. Previous studies on dislocations in $\mathrm{GaN}$ therefore focused on isolated aspects: Density-functional theory calculations [5,7] give an accurate description but are restricted to rather small system sizes (a few hundred atoms) thus excluding part of the strain effects. Empirical potentials [e.g., Stillinger-Weber (SW)-type potentials] permit large scale calculations with $10^{5}$ atoms; thus strain effects can be included, but the accuracy particularly near the core region is rather limited $[12,13]$. Finally, continuum elastic theory gives the correct asymptotic limits but not an atomic description at all.

To overcome these deficiencies we have applied a multiscale approach which combines elements of all three approaches and which allows one to study a system describing a dislocation and its strain field, i.e., a system consisting of up to a few $10^{5}$ atoms, with ab initio accuracy. A detailed description will be given elsewhere [14]. Here only key ideas of the approach are discussed.

The dislocations are described in a supercell geometry. Each supercell contains two edge dislocations with Burgers vectors of opposite sign. Based on the experimental data only threading dislocations with a core parallel to the [0001] axis and in the neutral charge state are considered. The dislocations are arranged in a "quadrupolar" configuration to ensure full symmetry (i.e., the net forces on each dislocation cancel) and to minimize dislocation-dislocation interaction [15]. In a first step linear elastic theory $[16,17]$ is used to construct the initial geometry. Specifically, the known relation of the displacement field for an isolated dislocation [16] is used to create by linear superposition the displacement field of a pseudoperiodic $12 \times 12$ dislocation array in quadrupolar configuration. The size of this array has been verified to be large enough to correctly describe the periodicity of a supercell.

In a second step the atomic structure of the supercell is created by mapping the displacement field onto the bulk structure. It is important to note that this procedure does not result in a unique atomic structure but that the structure depends on the choice of the origin of the dislocation within the bulk lattice. By choosing different values all previously studied structures of $\mathrm{GaN}$ dislocation cores have been constructed: the open- and full-core structures and their vacancy modifications [5]. In addition, we obtain also the already mentioned four-core structure which has so far not been considered by $a b$ initio studies [Fig. 2(a)]. Using this approach we have constructed a systematic set of supercells with dislocation-dislocation distances ranging between 21 and $350 \AA$. The smallest cell consists of 108 atoms the largest of $\approx 60000$ atoms.

In a third step we use Stillinger-Weber-type empirical potentials [18] to calculate total energy and atomic forces 
for all these structures. By relaxing the atomic geometry (until the forces on all atoms are $<10^{-3} \mathrm{eV} / \AA$ ) the (local) equilibrium configuration for each system is achieved. For the smaller cells (consisting of up to 144 atoms) equivalent calculations have been performed employing $a b$ initio density-functional theory. Specifically, the generalized-gradient approximation [19], a plane-wave basis set (energy cutoff $50 \mathrm{Ry}$ ), and soft TroullierMartins pseudopotentials [20] have been used. The Brillouin zone was sampled by a $1 \times 1 \times 2$ MonkhorstPack mesh [21]. Details about the method and convergence checks can be found elsewhere $[22,23]$.

In order to identify the energetically most favorable core structure we have calculated for all core structures the dislocation formation energy:

$$
E_{f}=\frac{1}{2 L_{z}}\left[E_{\mathrm{tot}}(\text { disl. })-E_{\mathrm{tot}}(\text { bulk })\right]
$$

Here, $E_{\text {tot }}($ disl.) is the total energy of the supercell containing the two dislocations after atomic relaxation, and $E_{\text {tot }}$ (bulk) is the total energy of GaN bulk consisting of the same number of atoms as the dislocation supercell. $L_{z}$ is the length of the supercell along the dislocation core (i.e., along [0001]), and the factor $1 / 2$ takes into account that there are two equivalent dislocations per supercell.

The relevant stability as a function of dislocationdislocation separation for all three edge dislocation struc-

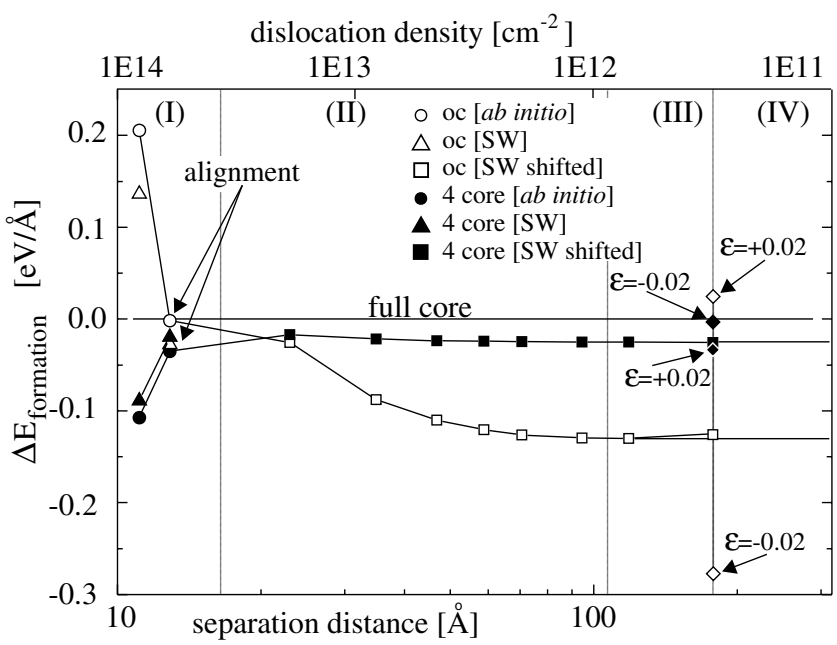

FIG. 3. Relative formation energies per dislocation as a function of dislocation separation (lower axis) and dislocation density (upper axis). The formation energy of the full-core structure has been used as reference energy. The symbols mark the calculated energies: ab initio calculations (cycles), shifted (squares), and unshifted empirical potentials (triangles). The open and filled diamonds give the relative formation energy if the dislocations are formed in a strained host. The value $\varepsilon$ gives the corresponding strain (positive/negative values indicate compressive/tensile strain). The vertical solid lines separate the four zones discussed in the text. Alignment shows the cell size for which the $a b$ initio and the empirical potential calculations have been aligned. tures (full, open, and four core) is shown in Fig. 3. The full-core structure is used as a reference. The figure contains two major pieces of information: (i) the slope of the energy dependence (which describes the defect-defect interaction) and (ii) the absolute value (which represents the energy of the inner core region). Since (i) is mainly realized by elastic interaction it should be well described by the empirical potentials. The inner core region, however, relevant for (ii) is characterized by broken bonds, a situation for which empirical potentials are commonly not well suited and ab initio calculations become mandatory. We therefore use the results for the largest $a b$ initio calculated supercell (the 144 atom cell) to shift for each dislocation type the empirical potentials. The shift is small [2 (24) meV/ $\AA$ for the full- (open-) core structure] indicating the high quality of the empirical potential.

In order to verify the accuracy of the multiscale approach Fig. 3 can be divided into four different regions. The first region (I) allows a direct comparison between $a b$ initio and empirical potential calculations. As can be seen the error is negligible $(0.069$ and $-0.018 \mathrm{eV} / \AA$ for the open- and the four-core structure, respectively) compared to the actual energy difference between the core structures. In the third region (III) the energy dependence becomes independent from the dislocation distance which is the expected behavior when linear elastic theory applies. This region connects empirical potentials with elastic theory and thus can be used to extrapolate the formation energy to experimentally relevant dislocation densities. Finally, the second region (II), accessible by empirical potential calculations only, bridges between the first (ab initio microscopic) and the third (experimentally relevant macroscopic) zone. The multiscale analysis (Fig. 3) clearly shows that for stoichiometric and neutral dislocations the open core is the most stable structure. This result is in contrast to the assignment based on the image simulations where the four-core structure had been identified (see Fig. 1). We have therefore tested the effect various mechanisms (such as charging the defect, passivation of dangling bonds by hydrogen, or strain) may have on the relative stability of the three investigated core structures. In order to test the dependence on strain we calculated the formation energy of the dislocations in large supercells (dislocation separation $\sim 177 \AA ; \approx 10^{5}$ atoms) where linear elastic theory applies for varying strain parameters. The relative energy of the open-core structure with respect to the full- and four-core structures dramatically changes when going from compressive to tensile strain: While the open-core structure is the most stable structure for compressive and zero strain conditions, the four-core structure becomes energetically most favorable for a tensile strain larger than $\approx 0.015$ (see Fig. 3). Actually, HVPE-grown material as investigated here is well known to be under significant tensile strain [24,25], and we therefore tentatively assign the stabilization of the four-core struc- 
ture as a consequence of significant tensile strain in the sample. Furthermore, the effect of $\mathrm{Ga}$ and $\mathrm{N}$ vacancies on the stability of the dislocation core has been considered. We find that for highly compensated $\mathrm{GaN}$ and medium to $\mathrm{N}$-rich growth conditions (as characteristic for HVPE) vacancy formation is highly endothermic. Under these conditions defect concentrations are too small to affect dislocation energetics or structure [14].

Using the results from the ab initio calculations we have analyzed the electronic character of the different dislocation cores. An analysis of the total density of states clearly showed that all cores have states in the band gap and are thus electrically active. This is the expected behavior for the open- and full-core structures since both have broken bonds in the core region. Indeed, the existence of these states has been reported also in previous density-functional theory studies [6,7]. For the four-core structure, however, which is completely reconstructed and where no broken bonds exist, this behavior is fully unexpected. In order to identify the mechanism inducing this state the corresponding defect wave function has been analyzed [Fig. 2(a)]. As can be seen the state is characterized by orbitals between two Ga atoms and similar to the states forming the metallic bonds in bulk $\mathrm{Ga}$. The origin of this state is (i) the large local strain field in the vicinity of the dislocation line and (ii) the small lattice constant characteristic for $\mathrm{GaN}$ (the lattice constant of GaAs, e.g., is larger by $20 \%$ ). Both effects result in a Ga-Ga separation of $2.74 \AA$ which is close to the bond length in bulk Ga (2.44 ̊) [26].

It is interesting to note that the strain-induced formation of empty bond states is not restricted to the four-core structure but appears to be a general feature of all $\alpha$-type dislocations in GaN [Figs. 2(a)-2(c)]. Indeed, recent experimental studies showed the appearance of states at an energy of $3.2 \mathrm{eV}$ above the valence band edge [27], which is in excellent agreement with the energetic position we find from our calculation. The main difference between the fully coordinated four-core structure and the other core structures is that in the latter case also the dangling bond orbitals clearly contribute to this state.

In conclusion, combining an ab initio based multiscale approach, HR-TEM, and image simulations, we have identified a novel core structure for dislocations in HVPE-grown GaN. Despite the fact that the core is fully reconstructed the electronic structure clearly shows the existence of deep dislocation related states. This result is in contrast to the classical picture of dislocations according to which the existence of deep states is related to the presence of broken bonds (and thus undercoordinated atoms) in the dislocation core. An analysis of the deep electronic states showed that they are related to the giant local strain field around a threading dislocation in $\mathrm{GaN}$ which is strong enough to induce a partial metallization in the form of metalliclike Ga-Ga bonds. It is crucial to note that in contrast to the classical picture, where deep electronic states can be removed by reconstructing the core, strain-induced deep states are rather insensitive to the specific core reconstruction and prevail even for fully reconstructed core structures.

Part of the TEM work has been performed in the Central Facility for High-Resolution Electron Microscopy of the Universität Erlangen. We thank A. Serra and P. Ruterana for fruitful discussions and acknowledge financial support from the EU TMR program IPAM. L. L. and J. N. acknowledge financial support from the DFG for the research group "Nitride-based QD lasers."

*Electronic address: neugebauer@fhi-berlin.mpg.de

[1] S.C. Jain, M. Willander, J. Narayan, and R. Van Overstraeten, J. Appl. Phys. 87, 965 (2000).

[2] S. S. Lester, F. A. Ponce, M. G. Crafod, and D. A. Steigerwald, Appl. Phys. Lett. 66, 1249 (1995).

[3] S. J. Rosner et al., Appl. Phys. Lett. 70, 420 (1997).

[4] J. Elsner et al., Phys. Rev. Lett. 79, 3672 (1997).

[5] A. F. Wright and U. Grossner, Appl. Phys. Lett. 73, 2751 (1998).

[6] S. M. Lee et al., Phys. Rev. B 61, 16033 (2000).

[7] C. J. Fall et al., Phys. Rev. B 65, 245304 (2002).

[8] M. Albrecht et al., J. Appl. Phys. 92, 2000 (2002).

[9] Y. Xin et al., Appl. Phys. Lett. 72, 2680 (1998).

[10] Y. Xin et al., Appl. Phys. Lett. 76, 466 (2000).

[11] See http://www.rz-berlin.mpg.de/ lymperak/hrtemDisl/ hrtem.htm

[12] J. Chen, P. Ruterana, and G. Nouet, Mater. Sci. Eng. B 82, 117 (2001)

[13] A. Béré and A. Serra, Phys. Rev. B 65, 205323 (2002).

[14] L. Lymperakis and J. Neugebauer (to be published).

[15] X. Blase et al., Phys. Rev. Lett. 84, 5780 (2000).

[16] J.P. Hirth and J. Lothe, Theory of Dislocations (McGraw-Hill, New York, 1968).

[17] M. Tu. Gutkin and E. C. Aifantis, Scr. Mater. 36, 129 (1996).

[18] F. H. Stillinger and T. A. Weber, Phys. Rev. B 31, 5262 (1985).

[19] J. P. Perdew, K. Burke, and M. Ernzerhof, Phys. Rev. Lett. 77, 3865 (1996); 80, 891 (1998).

[20] N. Troullier and J. L. Martins, Phys. Rev. B 43, 1993 (1991).

[21] H. J. Monkhorst and J. D. Pack, Phys. Rev. B 13, 5188 (1976).

[22] M. Bockstedte, A. Kley, J. Neugebauer, and M. Scheffler, Comput. Phys. Commun. 107, 187 (1997).

[23] C. D. Lee et al., Mater. Res. Soc. Symp. Proc. 743, L4.1.1 (2003).

[24] S. Hearne et al., Appl. Phys. Lett. 74, 356 (1999).

[25] T. Böttcher et al., Appl. Phys. Lett. 78, 1976 (2001).

[26] M. Bernasconi, G. L. Chiarotti, and E. Tosatti, Phys. Rev. B 52, 9988 (1995).

[27] U. Bangert et al., J. Appl. Phys. 93, 2728 (2003). 